\title{
Group 2 innate lymphoid cells protect lung endothelial cells from pyroptosis in sepsis
}

Dengming Lai ${ }^{1,2}$, Jing Tang $\mathbb{C}^{2,3}$, Linsong Chen ${ }^{2,4}$, Erica K. Fan ${ }^{5}$, Melanie J. Scott $\mathbb{C}^{2}$, Yuehua Li, ${ }^{2,6}$, Timothy R. Billiar ${ }^{2,7}$, Mark A. Wilson ${ }^{2,6}$, Xiangming Fang ${ }^{8}$, Qiang Shu' ${ }^{1}$ and Jie Fan ${ }^{2,6,7}$

\begin{abstract}
Group 2 innate lymphoid cells (ILC2) are one of three subgroups of innate lymphoid cells (ILC1, ILC2, and ILC3), and the major ILC population detected in the lungs. The function of ILC2 in the regulation of lung inflammation remains unclear. In the current study, we explored an important role of ILC2 in protecting lung endothelial cell (EC) from pyroptosis in sepsis-induced acute lung inflammation and the underlying mechanism. Using a cecal ligation and puncture (CLP) mouse sepsis model, we demonstrated that IL-33, which is released in response to sepsis, acting through its receptor ST2 mediates ILC2 expansion in the lungs. We further showed that the increased ILC2 in the lungs secrete IL-9, which in turn prevents lung EC from undergoing pyroptosis, a pro-inflammatory cell death form, by attenuating caspase-1 activation. These findings suggest a previously unidentified innate pathway that negatively regulates lung inflammation following sepsis.
\end{abstract}

\section{Introduction}

Sepsis is a life-threatening syndrome of organ dysfunction induced by dysregulated host responses to infection ${ }^{1}$. Although the hospital supportive care has been improved, sepsis is still a leading cause of mortality in the intensive care unit ${ }^{2-4}$. Mortality in sepsis is primarily due to multiple organ dysfunction syndrome, of which acute lung injury (ALI) is a common and important component ${ }^{5}$. At a cellular level, sepsis-induced endothelial dysfunction and injury of the pulmonary vasculature, especially the pulmonary microvasculature, are the predominant mechanisms for the development of ALI and are associated with higher mortality ${ }^{6,7}$.

Endothelial cells (ECs) serve as the sentinel cells for detecting infection and host defensing ${ }^{8}$. EC death triggered by lipopolysaccharide (LPS) and tumor necrosis

Correspondence: Xiangming Fang (xmfang@zju.edu.cn) or

Qiang Shu (shuqiang@zju.edu.cn) or Jie Fan (jif7@pitt.edu)

${ }^{1}$ Department of Thoracic and Cardiovascular Surgery, The Children's Hospital of

Zhejiang University School of Medicine, Hangzhou, Zhejiang, China

${ }^{2}$ Department of Surgery, University of Pittsburgh School of Medicine, Pittsburgh, PA, USA

Full list of author information is available at the end of the article Edited by H.-U. Simon factor $\alpha(\mathrm{TNF} \alpha)$ has been determined in septic mice ${ }^{9,10}$. Studies have shown that LPS through activating caspase-1 induces EC pyroptosis ${ }^{11}$, a caspase-1-dependent proinflammatory cell death type ${ }^{12}$. Cell pyroptosis, which can be triggered by various pathological stimuli, including infection, is characterized by plasma-membrane pore formation, intracellular content release, and DNA cleavage $^{12,13}$. Lung EC pyroptosis plays a significant role in the progression of $\mathrm{ALI}^{14}$.

Innate lymphoid cells (ILCs), a new member of the lymphoid population, play a central role in innate immunity of host response to inflammation, infection, and tissue damage. ILCs are further divided into three subgroups. ILC1 include natural killer (NK) and ILC1 cells, which produce interferon- $\gamma$. ILC2 produce type 2 cytokines, e.g., interleukin (IL)-9 and IL-13, and are dependent on transcription factors GATA-binding protein 3 (GATA3) and retinoic acid receptor-related orphan receptor- $\alpha$ (ROR- $\alpha)$ for development and function. ILC3 include all ILC subtypes that produce IL-17 and/or IL-22, and their development and function depend on the transcription factor ROR-yt ${ }^{15,16}$. ILC2 have been identified in both human and mouse lungs and airways, and 
these cells serve as a main ILC subtype in the lungs. ILC2 are also known to play an important role in maintaining airway barrier integrity and lung tissue homeostasis after virus infection ${ }^{16,17}$, and so have been suggested to be protective during infection ${ }^{17,18}$. However, the role of ILC2, as a major ILC population in the lungs, remains poorly characterized during sepsis and sepsis-induced acute lung inflammation.

Studies have suggested that epithelial or myeloid cellderived IL-25, IL-33, and thymic stromal lymphopoietin (TSLP) regulate ILC2 expansion and activation ${ }^{17,19-22}$. The expansion of tissue-resident ILC2 is followed by increased hematogenous emigration and redistribution of ILC2 under physiologic or pathological conditions ${ }^{23}$. Increased ILC2 in the lungs following sepsis was observed $^{24}$. However, the mechanism driving ILC2 into the lungs in sepsis remains unclear.

This study aims to elucidate the mechanism of ILC2 expansion in the lungs following sepsis, and the role of ILC2 in the regulation of lung inflammation during sepsis. We demonstrate here that IL-33, which is released mainly by epithelial cells in response to sepsis, acting through its receptor ST2 induces ILC2 expansion in the lungs. Increased ILC2 in the lungs secrete IL-9, which in turn prevents lung EC from undergoing pyroptosis by attenuating caspase- 1 activation. These findings suggest a previously unidentified pathway that negatively regulates lung inflammation following sepsis.

\section{Results}

Sepsis induces ILC2 expansion in the lungs and peritoneal cavity

Sepsis was induced in wild-type (WT) mice using a clinically relevant model of polymicrobial peritonitis caused by cecal ligation and puncture (CLP) ${ }^{25}$. ILC2 in the lungs at baseline and time points up to $36 \mathrm{~h}$ after CLP or sham surgery (SS) were detected by flow cytometry and defined as $\mathrm{Lin}^{-} \mathrm{CD} 45^{+} \mathrm{CD} 90.2^{+} \mathrm{ST} 2^{+}$cells (see gating strategy in Supplementary Fig. 1a) ${ }^{26}$. Additional ILC2 markers, including Sca-1, KLRG1, CD25, and CD127 were also used to further confirm ILC2 identification (Supplementary Fig. 1b) ${ }^{17,26}$. The percentage and absolute number of ILC2 in the lungs at $12 \mathrm{~h}$ after CLP was significantly increased as compared with that in SS mice, and the ILC2 number remained elevated for at least additional $24 \mathrm{~h}$ (Fig. 1a, b). The percentage and absolute number of ILC2 in the lungs of SS mice did not show significant changes.

ILC2 in the peritoneal lavage fluid (PLF) were also measured after CLP. As shown in Fig. 1c, d, CLP induced an increase in the percentage and absolute number of peritoneal ILC2, which peaked at $24 \mathrm{~h}$, but remained significantly elevated at $36 \mathrm{~h}$ after CLP.

These data indicate that ILC2 increase in the lungs and peritoneal cavity in response to CLP-induced sepsis.

\section{IL-33/ST2 signaling is required for sepsis-induced ILC2 expansion}

TSLP, IL-25, and IL-33 were previously suggested to play a role in mediating ILC2 expansion and activation ${ }^{17,19-22}$. We therefore measured Tslp, Il-25, and Il-33 mRNA expression, using Real-time PCR, in the lungs of CLP mice for up to $24 \mathrm{~h}$; and found that the expression of all these cytokines were significant increased and peaked at $6 \mathrm{~h}$ after CLP (Fig. 2a). To determine whether these cytokines mediate sepsis-induced ILC2 recruitment in the lungs, we intratracheally (i.t.) injected normal WT mice with recombinant mouse TSLP (rmTSLP), IL-25 (rmIL-25), IL33 (rmIL-33), or phosphate-buffered saline (PBS; control), and assessed percentage and absolute number of ILC2 in the lungs at $24 \mathrm{~h}$ after the injection. Treatment with rmIL33 induced significant increase of ILC2 in the lungs as compared with PBS control group (Fig. 2b, c). It is noticeable that the number of ILC2 in the lungs induced by IL-33 is similar to that induced by CLP at $24 \mathrm{~h}$. Treatment with rmIL-25 also increased the percentage and number of ILC2 in the lungs as compared with PBS-treated group, but the levels were significantly lower than that in the rmIL33-treated mice and CLP mice (Fig. 2b, c). Despite the increase in Tslp mRNA expression in the lungs after CLP, treatment with rmTSLP did not increase the percentage and number of ILC2 in the lungs as compared with PBStreated group (Fig. 2b, c).

To confirm the role of IL-33 in mediating ILC2 expansion in the lungs, we first detected IL-33 receptor ST2 expression on the ILC2. Since the Sca-1 and KLRG-1 double-positive in lineage-negative cells were nearly $100 \%$ GATA3-positive and has been defined as ILC2 ${ }^{27}$, we used the strategies by measuring the cell markers of Sca-1 and KLRG-1 on $\mathrm{Lin}^{-} \mathrm{CD} 45^{+} \mathrm{CD} 90.2^{+} \mathrm{ST} 2^{+}$cells and the expression of ST2 and CD90.2 on $\mathrm{Lin}^{-} \mathrm{CD} 45^{+} \mathrm{Sca}-1^{+}$cells to define the ST2 expression on ILC2. We found that more than $90 \%$ of $\mathrm{Lin}^{-} \mathrm{CD} 45^{+} \mathrm{CD} 90.2^{+} \mathrm{ST} 2^{+}$cells express Sca- 1 and KLRG-1, and $~ 99 \%$ of Lin ${ }^{-}$CD $45^{+}$Sca- $1^{+}$cells express ST2 and CD90.2 (Fig. 2f). We then subjected Il$33^{-/-}$mice and $I l 1 r l 1^{-/-}$mice (genetic deficiency of ST2) to CLP and measured the percentage and number of $\mathrm{Lin}^{-} \mathrm{CD} 45^{+} \mathrm{Sca}-1^{+}$cells, which were also defined as $\mathrm{ILC} 2^{24}$, in the lungs at $24 \mathrm{~h}$ after CLP. We demonstrated that deficiency of either Il-33 or Il1rl1 prevented ILC2 expansion in the lungs following sepsis (Fig. 2d, e, g, h). Moreover, i.t. administration of rmIL-33 to $\mathrm{Il}-33^{-/-}$mice restored the increase of ILC2 in the lungs at $24 \mathrm{~h}$ after CLP and even SS (Fig. 2d, e). Collectively, these results strongly indicate a role for IL-33/ST2 signaling in ILC2 expansion in the lungs following sepsis.

\section{ILC2 protect lung EC from death following sepsis}

EC death plays a significant role in pulmonary microvascular dysfunction associated with ALI following 


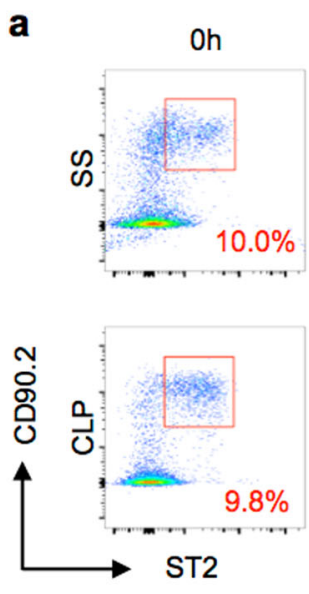

c
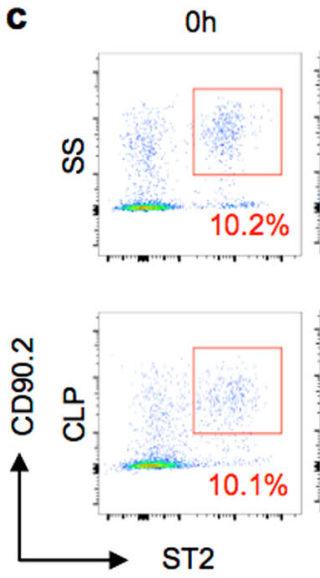

$12 \mathrm{~h}$
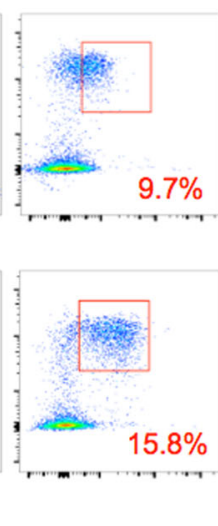

$12 \mathrm{~h}$
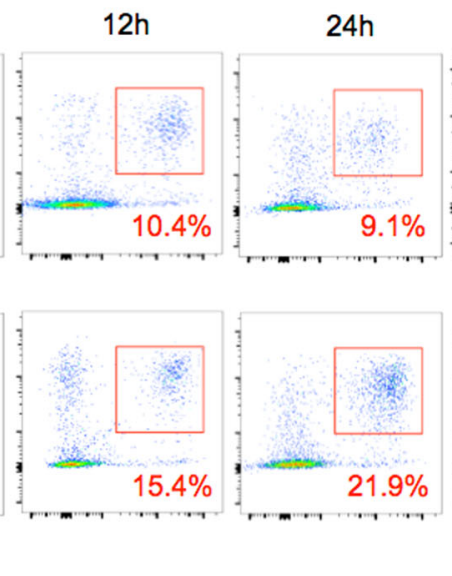
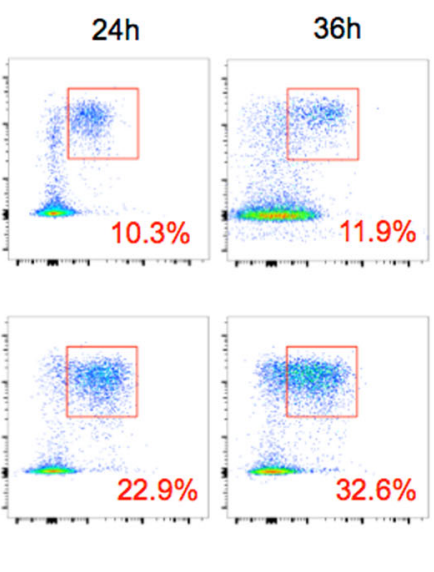

$36 \mathrm{~h}$

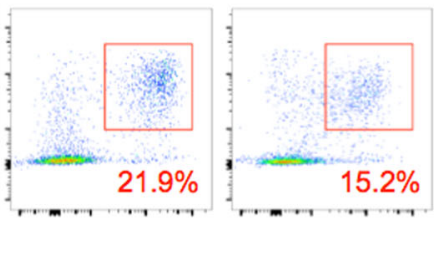

b

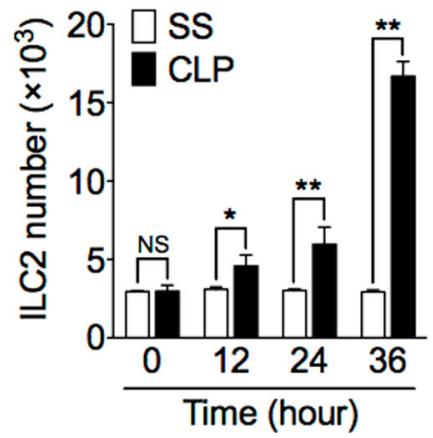

d

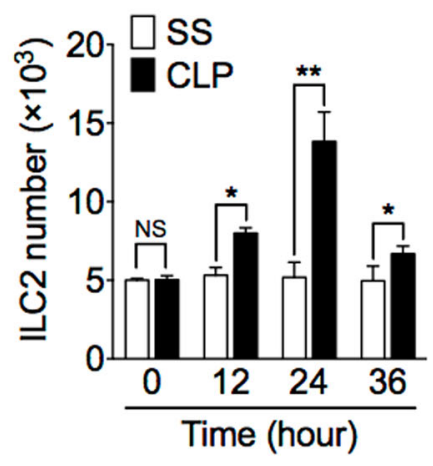

Fig. 1 Sepsis induces ILC2 expansion in lungs and peritoneal cavity. Representative flow cytometry plots showing percentages of $\mathrm{Lin}^{-} \mathrm{CD} 45^{+} \mathrm{CD} 90.2^{+} \mathrm{ST} 2^{+} \mathrm{ILC} 2$ in a lung and $\mathbf{c}$ peritoneal lavage fluid of WT mice at 0, 12, 24, and $36 \mathrm{~h}$ after CLP or sham surgery (SS). Bar graphs showing ILC2 absolute cell number in $\mathbf{b}$ lung and $\mathbf{d}$ peritoneal cavity in SS or CLP mice at time points up to $36 \mathrm{~h}$. $n=6 \mathrm{mice} / \mathrm{group}$. Data are shown as mean \pm SEM. ${ }^{*} P<0.05,{ }^{* *} P<0.01$, NS not significant

sepsis ${ }^{9}$. Lung ILC2 have been suggested to play a role in restoring airway integrity and lung tissue homeostasis ${ }^{17}$. These findings, therefore, led us to ask whether lung ILC2 involve in maintaining the integrity of lung EC in sepsis. We subjected WT and $I l-33^{-1-}$ mice to CLP and measured mouse lung EC (MLEC) death using Annexin V/7AAD staining detected by flow cytometry. As shown in Fig. 3a, b, CLP markedly induced MLEC death in $\mathrm{Il}-33^{-/-}$ mice as compared with that in WT mice.

To determine a causal relationship between the decreased ILC2 in the lungs and increased MLEC death in Il-33 ${ }^{-/-}$mice following sepsis, we co-cultured ILC2 with mouse MLEC in vitro in the presence and absence of LPS and TNF $\alpha$, which mimic sepsis stimulation. As shown in Fig. 3c, d, co-culture with ILC2 markedly reduced MLEC death induced by LPS and TNF $\alpha$, suggesting a direct protective effect of ILC2 on MLEC in sepsis. However, addition of rmIL-33 in the culture medium failed to protect MLEC death (Fig. 3e, f).
To further determine the impact of MLEC death on lung vascular leaking, we applied Evans blue dye (EBD) to assess blood vessel permeability in vivo. We demonstrated that at $24 \mathrm{~h}$ after CLP, the lungs of $\mathrm{Il}-33^{-/-}$mice had increased permeability shown as more blue coloration in the lungs than that in WT lungs (Fig. 3g). The EBD extravasation was also quantified. As shown in Fig. 3h, EBD concentration was twofold higher in the lungs of $\mathrm{Il}$ $33^{-/-}$mice than that in WT mice.

These data demonstrated that IL-33-mediated ILC2 expansion in the lung prevents MLEC from death, but not IL-33 itself.

\section{Characterization of cytokine expression and secretion from lung ILC2 in sepsis}

Studies have shown that ILC2 are an important cellular source of type 2 cytokines ${ }^{17,19,28}$. To characterize the type 2 cytokines secreted from lung ILC2 following sepsis, we measured IL-4, IL-9, and IL-13 expression in the ILC2 


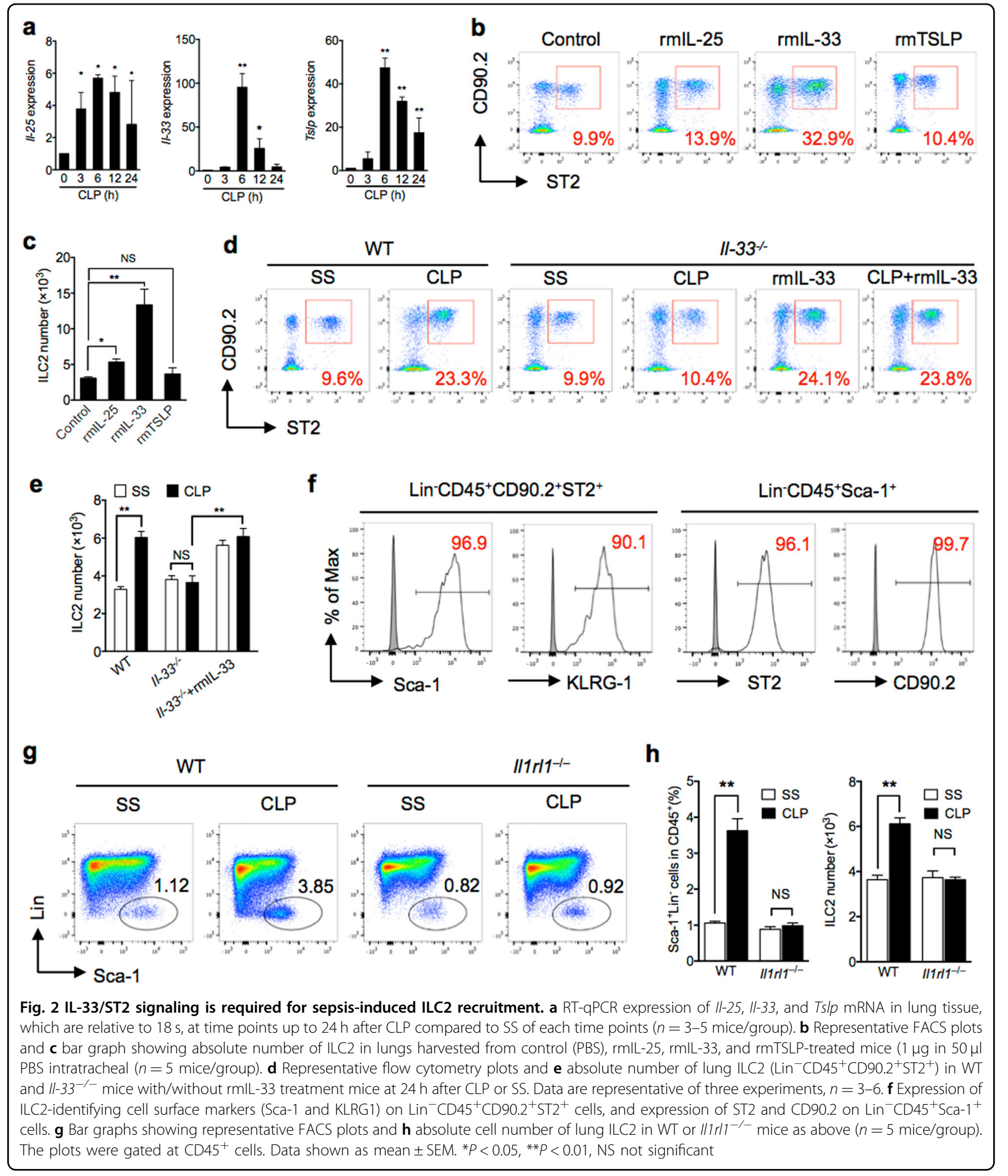

increased in the lungs by flow cytometry. We found that IL-9 positive (IL-9 ${ }^{+}$) ILC2 significantly increased by $24 \mathrm{~h}$ after CLP, and IL- $13^{+}$ILC2 transiently increased after CLP and reached a peak at $6 \mathrm{~h}$ (Fig. $4 \mathrm{a}, \mathrm{b})$. IL-4 ${ }^{+}$ILC2, however, did not significantly increase following sepsis
(Fig. 4a, b). We then measured plasma concentrations of IL-9 and IL-13 following sepsis, and demonstrated that both cytokines were markedly increased between 12 and $24 \mathrm{~h}$ after CLP (Fig. 4c). However, both $\mathrm{Il}-33^{-/-}$and Illrll ${ }^{-/}$mice exhibited reduced levels of IL-9 and IL-13 
a

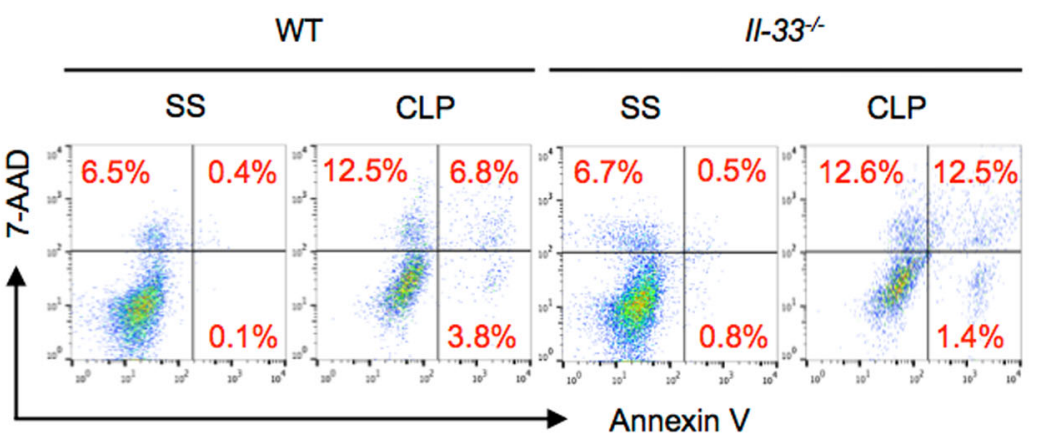

c

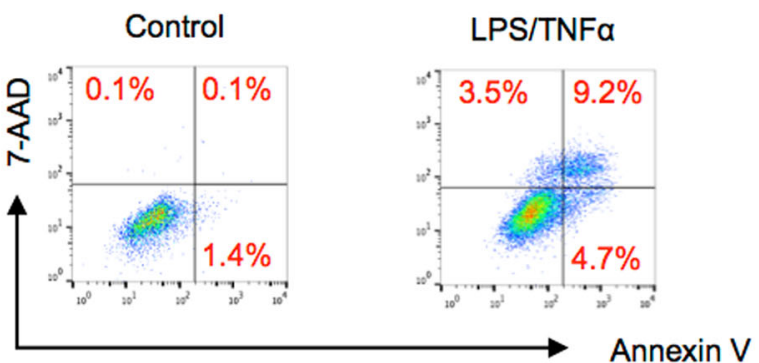

e

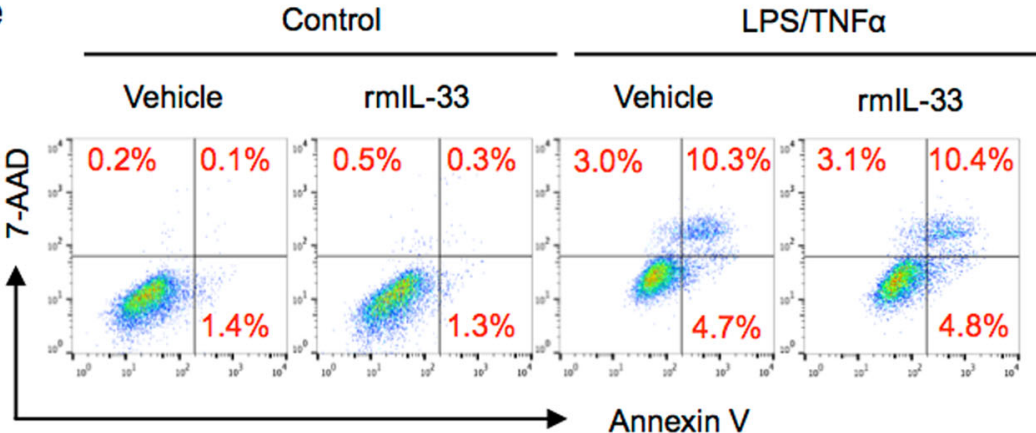

b

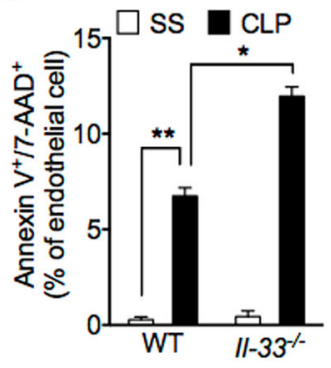

d
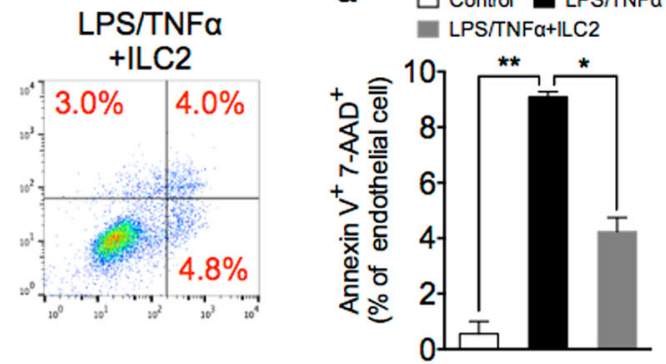

f

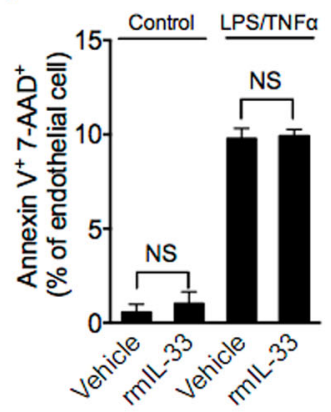

g

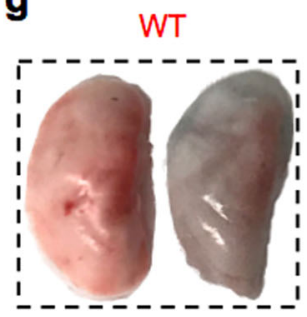

SS

CLP

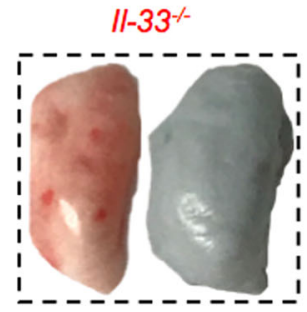

SS CLP h

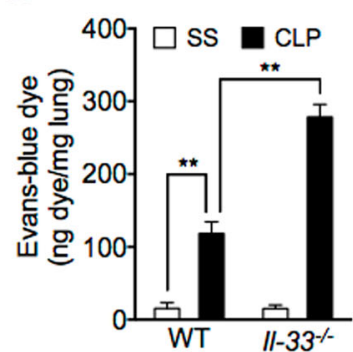

Fig. 3 ILC2 protect lung EC from death following sepsis. a Representative flow cytometry plots of Annexin V/7-AAD staining of MLEC from WT and $11-33^{-1-}$ mice at $24 \mathrm{~h}$ after SS or CLP. MLECS were identified as CD31 $1^{+}$and Annexin V/7-AAD double-stained cells were analyzed as dying $(n=5$ mice/group). $\mathbf{b}$ Bar graph of Annexin V/7-AAD double-stained cells as a percentage of MLEC in WT and $11-33^{-1-}$ mice at $24 \mathrm{~h}$ after SS or CLP. $\mathbf{c}$ Representative flow cytometry plots of Annexin V/7-AAD staining of in vitro primary isolated MLEC from WT mice cultured alone (control), with LPS $(1 \mathrm{\mu g} / \mathrm{ml})+$ TNFa $(20 \mathrm{ng} / \mathrm{ml})$, or LPS + TNFa + co-culture with ILC2 $\left(1 \times 10^{4}\right.$ cells/well $)$ for $24 \mathrm{~h}$. $\mathbf{d}$ Bar graph of Annexin V/7-AAD double-stained cells as a percentage of total MLEC. $\mathbf{e}$ Representative flow cytometry plots and $\mathbf{f}$ bar graph of Annexin V/7-AAD staining of purified MLEC from WT mice cultured with LPS $(1 \mathrm{\mu g} / \mathrm{ml})+$ TNFa $(20 \mathrm{ng} / \mathrm{ml})$ and with or without $\mathrm{rmlL}-33(50 \mathrm{ng} / \mathrm{ml})$ for $24 \mathrm{~h}$. g Representative images of whole lungs stained with Evans blue dye from WT and $11-33^{-1-}$ mice at $24 \mathrm{~h}$ following SS or CLP. $\mathbf{h}$ Colorimetric quantitative analysis of Evans blue dye extracted from stained lungs from WT and $11-33^{-1-}$ mice at $24 \mathrm{~h}$ following SS or CLP ( $n=5$ mice/group). Data are representative of three independent in vitro experiments. Data shown are the mean \pm SEM. ${ }^{*} P<0.05,{ }^{* *} P<0.01$ 


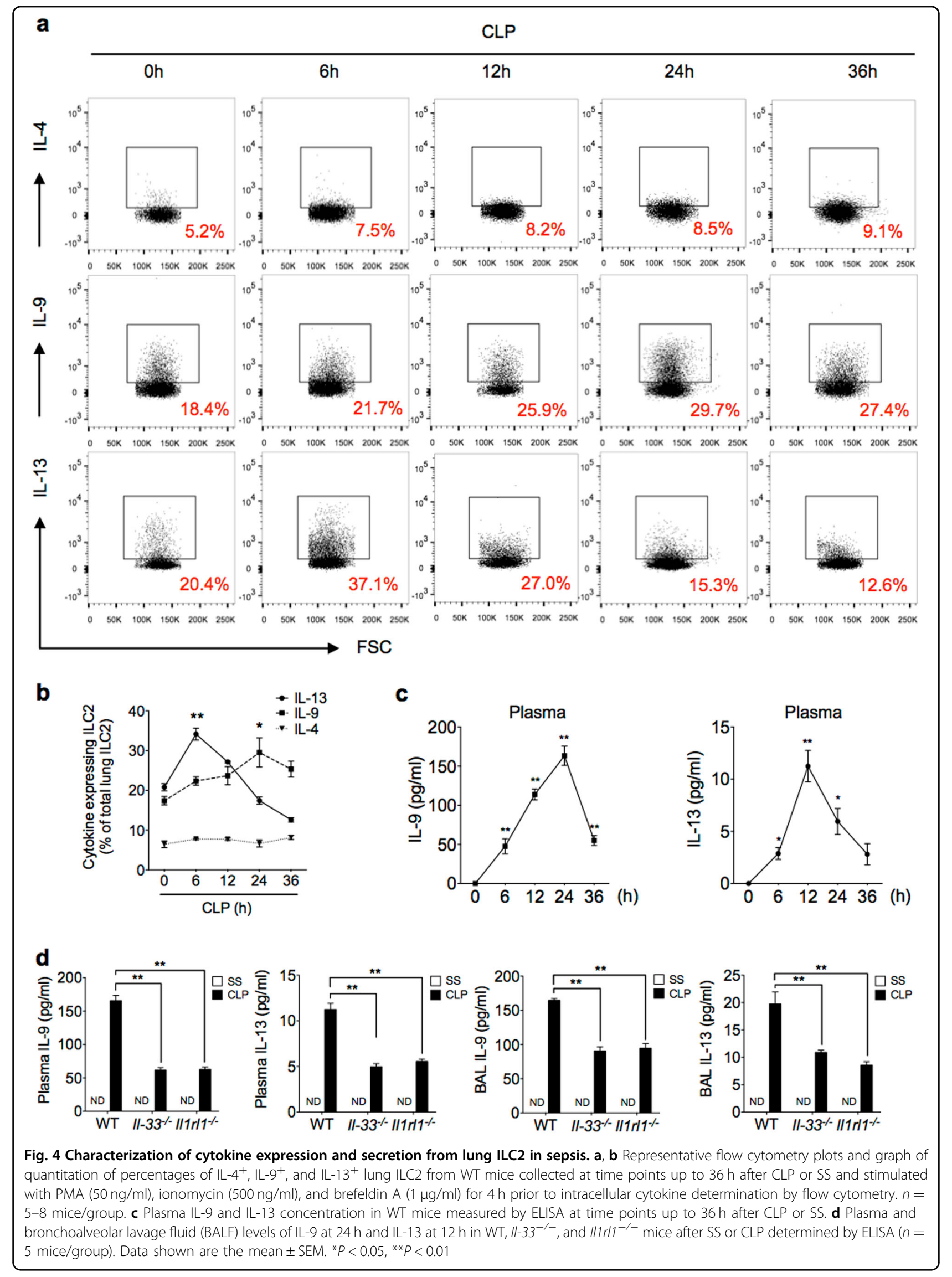


in plasma and bronchoalveolar lavage fluid (BALF) after sepsis as compared with those in WT mice (Fig. 4d). These results suggest a specific pattern of cytokine expression and secretion from lung ILC2, which increased in the lungs following sepsis in an IL-33/ST2-dependent manner.

\section{ILC2-derived IL-9 protects MLEC from pyroptosis}

IL-9 has recently been reported as a regulator in the pathogenesis of many inflammatory diseases ${ }^{29,30}$. For example, IL-9 is involved in protective immunity to Trichuris muris infections ${ }^{31}$, promotes Treg activation and prevents excessive cartilage destruction and bone loss in arthritis $^{32}$, and promotes tissue repair by amplifying ILC2 function in helminth-induced lung inflammation ${ }^{33}$. In lung tissue, IL-9 production was restricted to IL-33induced ILC2 in physiological or inflammatory conditions $^{34}$. IL-9 induces vascular endothelial adhesion molecule-1 expression on mouse aortic EC and attenuates lung inflammation ${ }^{29,33,35}$. We hypothesized that IL-9 might mediate the ILC2-dependent MLEC protection in sepsis. To test this hypothesis, we first determined whether MLECs express cell surface IL-9 receptor (IL-9R). As shown in Supplementary Fig. 2a, MLECs express a considerable level of IL-9R. We then treated MLECs with LPS and TNF $\alpha$ in vitro for $24 \mathrm{~h}$ in the presence and absence of rmIL-9 or rmIL-13, and found that rmIL-9 significantly reduced MLEC death; whereas, rmIL-13 failed to protect the MLEC from death (Fig. 5a, b). Furthermore, neutralizing antibody against IL-9 was added into the ILC2MLEC co-culture system, which challenged with LPS and TNF $\alpha$, and demonstrated that ILC2 no longer protect the MLEC from death (Fig. 5c, d).

We further defined the MLEC death type by staining the cells with TUNEL and Alexa Fluor 488-labeled caspase-1 FLICA following LPS and TNF $\alpha$ challenge. We found that TUNEL and activated caspase-1 double-positive MLECs, which represent pyroptosis, occupied $\sim 70 \%$ of the MLEC death (Fig. 5e, f), and IL-9 reduced MLEC pyroptosis by $\sim 60 \%$ (Fig. 5e, f).

To further confirm the observed MLEC death is dependent on caspase-1 activation, we treated MLEC from Caspase- $1^{-/-}$mice with LPS and TNF $\alpha$ for $24 \mathrm{~h}$ and measured the level of cell death. Figure $5 \mathrm{~g}$, h shows that caspase- 1 deficiency reduced MLEC death by $\sim 70 \%$.

Taken together, these results suggest that ILC2-derived IL-9 mediates the protection of MLEC from pyroptosis.

\section{IL-9 decreases caspase-1 activation in lung EC}

To determine whether IL-9 attenuates MLEC pyroptosis through suppressing caspase-1 activation, we treated the MLEC with LPS and TNF $\alpha$ for $24 \mathrm{~h}$ in the presence and absence of rmIL-9, and visualized caspase-1 activation and nuclear fragmentation by staining the cells with Alexa Fluor 488-labeled caspase-1 FLICA and TUNEL in the MLEC and observed using confocal microscopy. As shown in Fig. 6a, LPS and TNF $\alpha$ induced caspase- 1 activation and nuclear fragmentation in the MLEC, whereas rmIL-9 decreased caspase- 1 activation in the MLEC. Intracellular caspase-1 activation was also measured in the MLEC by flow cytometry and western blotting. IL-9 exhibited a significant suppressive effect on caspase- 1 activation in the MLEC in response to LPS and TNF $\alpha$ (Fig. 6b, c). To confirm the protective role of IL-9 in CLP-induced MLEC death, we injected the CLP mice (i. t.) with neutralizing antibody against IL-9, and found that the IL-9 antibody increased MLEC death following CLP (Fig. 6d, e). Altogether, these data show a novel role for ILC2-derived IL-9 in protection of MLEC from pyroptosis through suppressing caspase-1 activation.

\section{Discussion}

A number of studies have identified ILC2 in lung tissue and implicated these cells in the regulation of immunity and inflammation in the lung ${ }^{17}$. The data presented here show that IL-33/ST2 signaling leads to a significant expansion of ILC2 cells in the lungs and peritoneal cavity following CLP-induced sepsis. Expansion of ILC2 in the lungs provides ILC2-derived IL-9 in the lungs, which reduces sepsis-induced EC pyroptosis through suppressing caspase- 1 activation. These findings represent a novel pathway that regulates lung EC death and thereby affecting the progression of acute lung inflammation following sepsis.

Epithelial, endothelial, and myeloid cells in most organs express IL-25, IL-33, and TSLP, these are known to modulate ILC2 expansion in the lungs and activation in inflammation ${ }^{17,19-22,36}$. In the current study, we show that IL-25, IL-33, and TSLP expression increased in response to systemic inflammation caused by experimental peritonitis, and sepsis induces expansion of ILC2 in the lungs consistent with the changes in the expression of IL-25, IL33 , and TSLP. However, it was only the absence of IL-33, and its receptor ST2, that the increase in lung ILC2 cells was abolished and type 2 cytokines, including IL- 9 and IL13 were decreased, and rmIL-33 could rescue the phenotype, highlighting a role for IL-33 in ILC2 expansion and activation ${ }^{22}$.

EC death has been reported to play an important role in activation of a number of inflammatory cells, release of inflammatory mediators, and induction of inflammation in sepsis $^{37}$. Previous report showed that EC death occurs in sepsis $^{38}$, and TNF superfamily-associated pathways and activated caspases mediate the endothelial death ${ }^{10,39}$. We also observed lung EC death, as defined with the positive markers of CD31, Annexin V, and 7-AAD, following sepsis. Recently, IL-9 has been reported to be involved, either beneficially or deleteriously, in the pathogenesis of some 
a

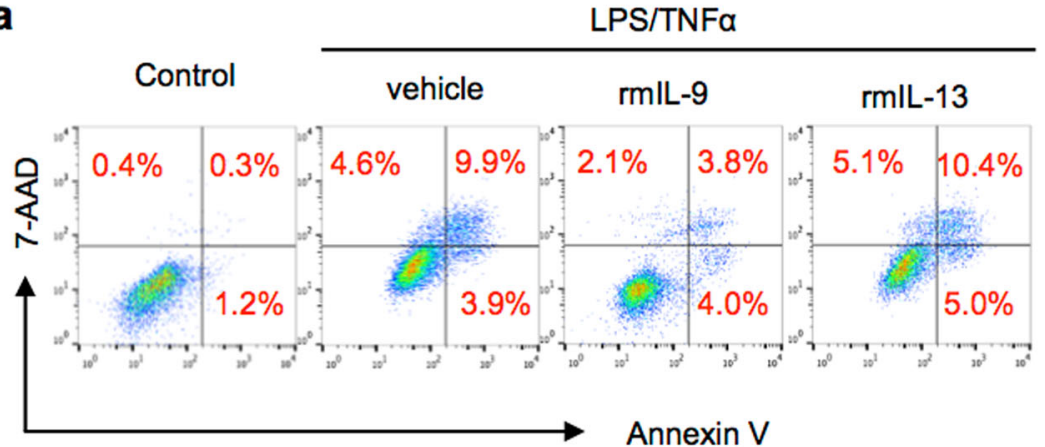

c

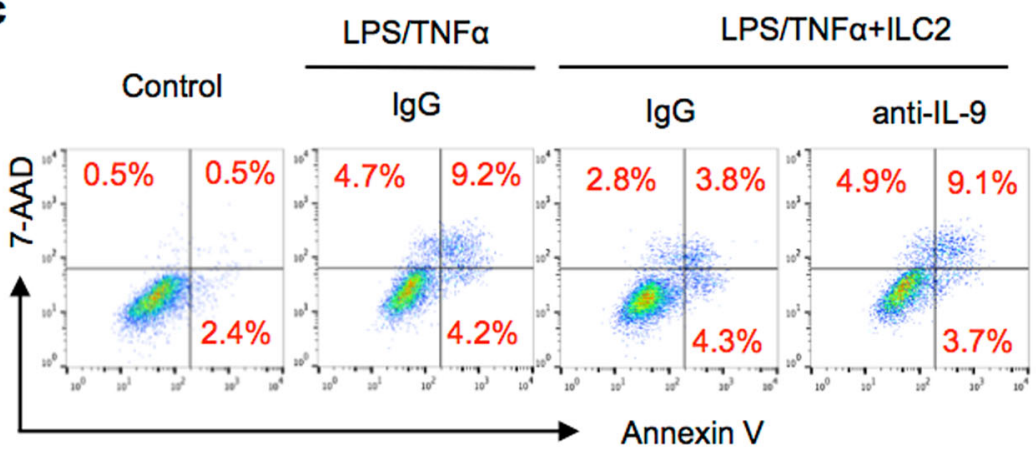

e

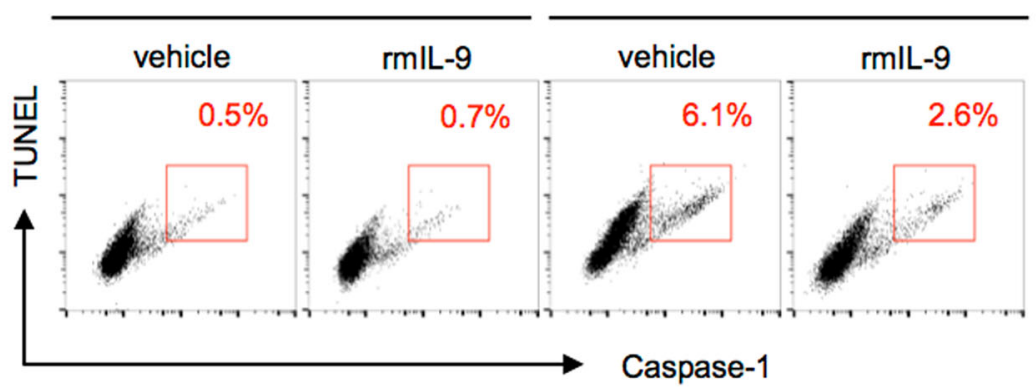

g

WT MLEC

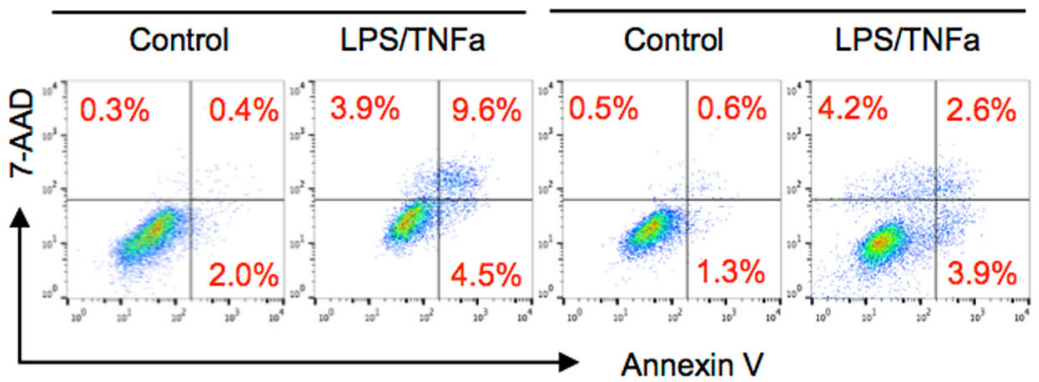

b

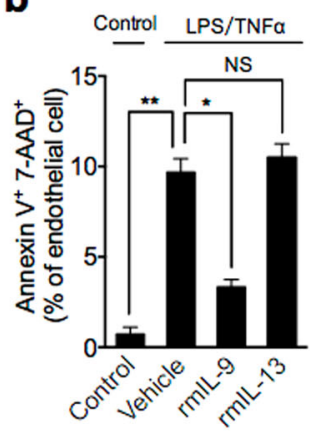

d

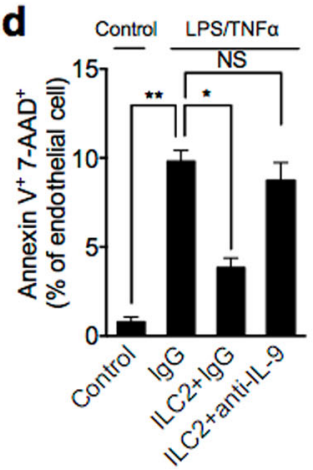

f

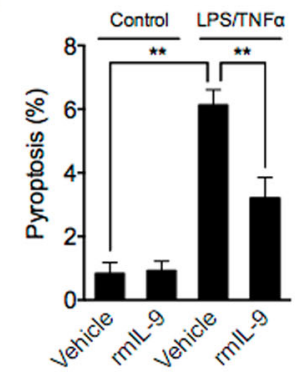

h

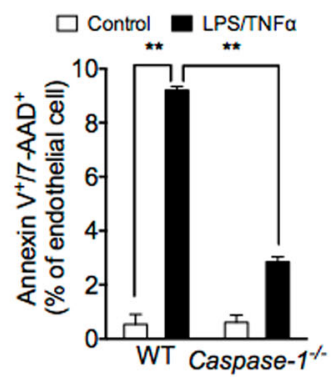

Fig. 5 ILC2-derived IL-9 protects MLEC from pyroptosis. $\mathbf{a}$, b Representative flow cytometry plots and quantitation of Annexin V/7-AAD staining of MLEC cultured with LPS/TNFa and with or without rmlL-9 or rmlL-13 for $24 \mathrm{~h}$. $\mathbf{c}$, $\mathbf{d}$ Representative flow cytometry plots and quantitation of Annexin V/7-AAD staining of MLEC co-cultured with ILC2 $\left(1 \times 10^{4}\right.$ cells/well) and LPS $(1 \mu \mathrm{g} / \mathrm{ml})+$ TNFa $(20 \mathrm{ng} / \mathrm{ml})$ and with lgG or anti-IL-9 for $24 \mathrm{~h}$. e, $\mathbf{f}$ Representative flow cytometry plots and quantitation of MLEC pyroptosis (caspase-1/TUNEL double-positive cells) with rmIL-9 (50 ng/ml), LPS, and TNFa for 24 h. g, h Representative flow cytometry plots and quantitation of Annexin V/7-AAD staining of MLEC from WT or Caspase- ${ }^{-1-}$ mice with LPS/TNFa for $24 \mathrm{~h}$. MLECs were identified as $C D 31^{+}$cells. Data shown are the mean $\pm \mathrm{SEM}, n=3-6$ mice/group. ${ }^{*} P<0.05$, ${ }^{* *} P<0.01$ 
a

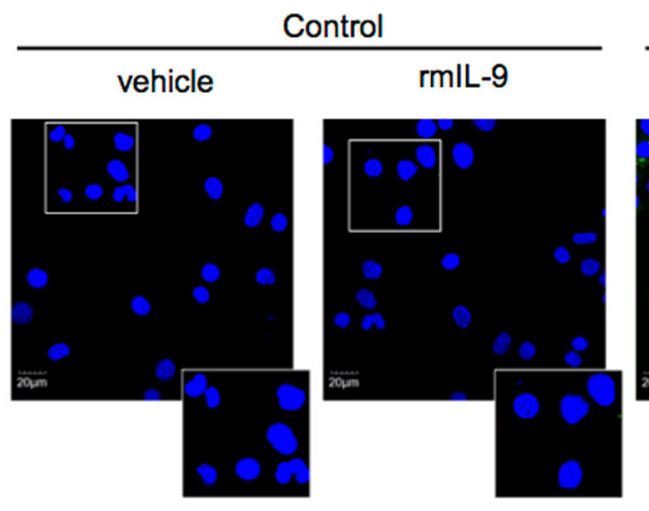

Caspase-1 TUNEL Hoechst

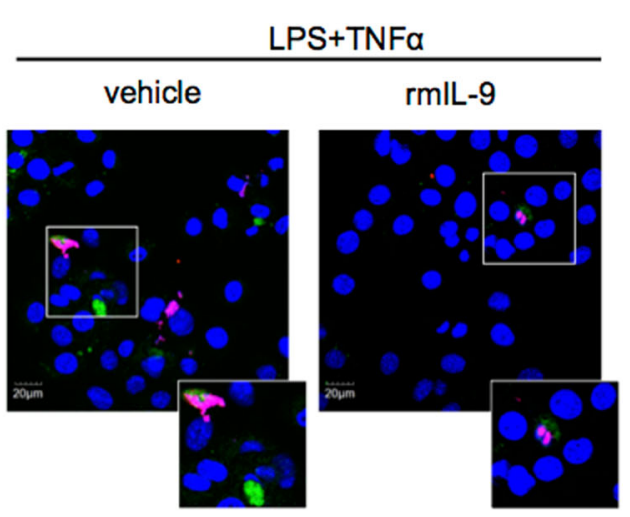

5

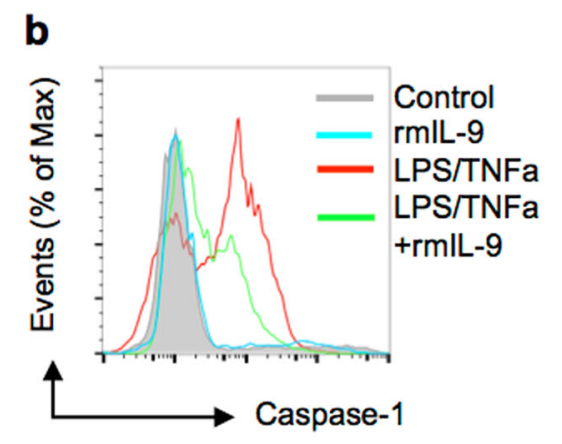

b

d

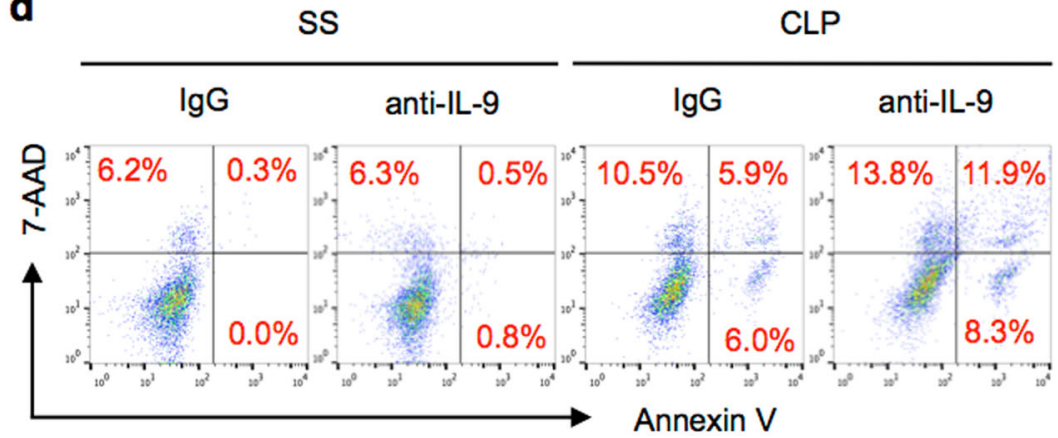

C
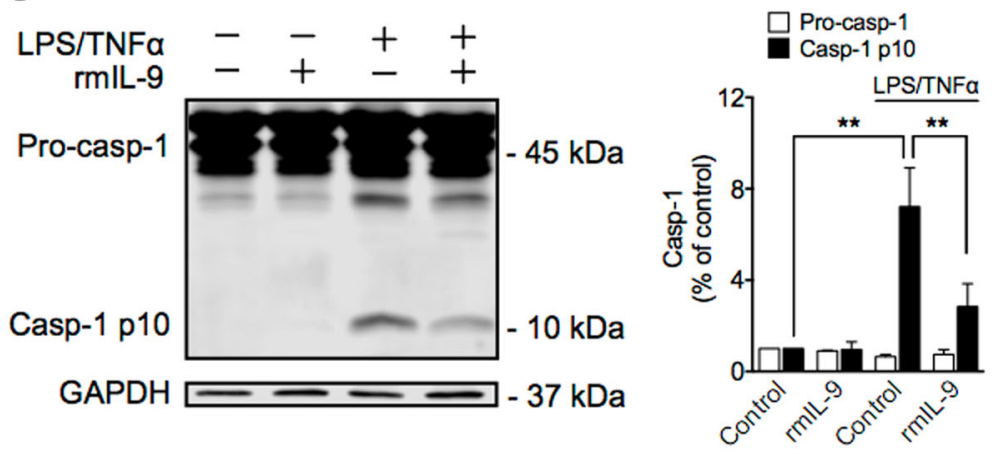

e

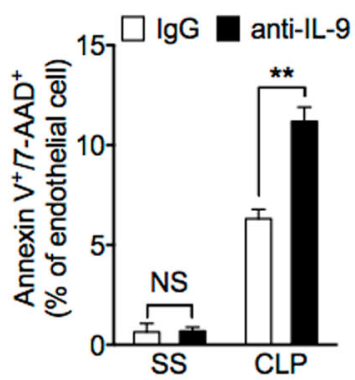

Fig. 6 IL-9 decreases caspase-1 activation in lung EC. a Confocal microscopy immunofluorescence of MLEC stained for activated caspase-1 (green), TUNEL (red), and Hoescht (blue) at $24 \mathrm{~h}$ cultured with rmlL-9 (50 ng/ml), LPS, and TNFa. b Number of MLEC expressing activated caspase-1 at $6 \mathrm{~h}$ after treatment with LPS and TNFa with or without rmlL-9 (50 ng/ml). c Western blots of pro-caspase-1 (Pro-casp-1) and activated/cleaved caspase-1 (Casp-1 p10) in whole-cell lysates of MLEC at $6 \mathrm{~h}$ after LPS, TNFa, and/or rmlL-9 (50 ng/ml). d Representative flow cytometry plots of MLEC from WT mice treated with nonspecific lgG or anti-IL-9 antibody (1 mg/kg) at 30 min before SS or CLP. MLECS were isolated at $24 \mathrm{~h}$ after CLP, and identified as $\mathrm{CD}_{1}{ }^{+}$, and Annexin V/7-AAD double-stained cells were analyzed as dying ( $n=5$ mice/group). e Bar graph of Annexin V/7-AAD doublestained cells as demonstrated in $\mathbf{d}$. Data shown are the mean $\pm \mathrm{SEM}, n=3-6$ mice/group in vivo. ${ }^{*} P<0.05,{ }^{* *} P<0.01$

diseases related to inflammation ${ }^{29,30}$. ILC2 is the main source of IL-9 in mouse lung tissue in physiological or inflammatory circumstances ${ }^{24}$. Our data showed that genetic deficiency of $I l-33$ prevented ILC2 expansion in the lung and increased MLEC death following CLP-induced sepsis; and addition of IL-33 into MLEC did not affect MLEC death in response to LPS and TNF $\alpha$. However, co- culture of MLEC with ILC2 significantly decreased MLEC death. Meanwhile, neutralization of IL-9 with anti-IL-9 antibody diminished the beneficial effect of ILC2. Furthermore, restoring IL- 9 by addition of IL- 9 into the cocultures decreased the LPS/TNF $\alpha$-induced MLEC death. These results indicate a protective role for ILC2-derived IL9 in protecting lung EC from death following sepsis. 
Cell death plays a significant role in inflammation and infection. The types of cell death can be non-programmed or programmed. Programmed cell death includes apoptosis, autophagy, necrosis, necroptosis, and pyroptosis. Pyroptosis is a caspase-1-dependent form of cell death ${ }^{12}$, which characterized by pore formation in the plasma membrane, cell swelling, and DNA cleavage ${ }^{40}$. Thus, pyroptotic cell should be Annexin V and propidium iodide double-positive ${ }^{41}$. In our study, we have observed Annexin $\mathrm{V}^{+} 7-\mathrm{AAD}^{+} \mathrm{EC}$ in lung after CLP. And then we determined that IL-9 protects EC from pyroptosis in vitro by caspase- 1 and TUNEL staining. The death of Caspase- $1^{-1-}$ MLEC was reduced by $\sim 70 \%$, as compared with WT MLEC, indicating that caspase-1-dependent pyroptosis serves as a major form of MLEC death following sepsis. To address the mechanism of IL-9 protect EC from pyroptosis, we determined the caspase-1 activation. ILC2-derived IL-9, as shown in the data, through attenuating caspase- 1 activation in MLEC reduces MLEC pyroptosis in sepsis.

In summary, the current study reveals a previously unidentified mechanism by which innate pathway negatively regulating lung inflammation following sepsis. The mechanism includes the regulation of enhanced ILC2 expansion in the lung and the protective role of ILC2derived IL-9 in reducing lung EC pyroptosis.

\section{Materials and methods Mice}

Male C57BL/6J WT mice were purchased from Jackson Laboratories (Bar Harbor, ME). Il-33-deficient $\left(\mathrm{Il}-33^{-/-}\right.$) and Il1rl1-deficient $\left(\mathrm{IlIrl1}^{-1-}\right.$, deficiency of ST2) mice were bred and maintained under specific pathogen-free conditions at the Animal Facility of the University of Pittsburgh School of Medicine. All mice were on a C57BL/ 6 genetic background and were carried out with 8-weekold male mice according to the guidelines of the Institutional Animal Care and Use Committee of the University of Pittsburgh, VA Pittsburgh Healthcare System.

\section{CLP model}

Sepsis was induced by CLP model as described previously $^{25}$. Briefly, mice were anaesthetized by intraperitoneal administration of ketamine $(50 \mathrm{mg} / \mathrm{kg})$ and xylazine $(5 \mathrm{mg} / \mathrm{kg})$. A double puncture was made through the cecum with an 18-gauge needle. A small amount of feces was gently squeezed out of the perforation sites to ensure patency of punctures. The cecum was then relocated into the abdominal cavity, and the laparotomy was closed. Mice received a subcutaneous injection of $1 \mathrm{ml}$ PBS immediately after surgery for fluid resuscitation, and then were returned to their home cages. At the study end point, mice were sacrificed, BALF, PLF, and lung tissue were collected under sterile conditions, and blood was withdrawn by cardiac puncture.

\section{Treatment of mice}

Mice were treated i.t. with $1 \mu \mathrm{g}$ recombinant murine IL33 (BioLegend, San Diego, CA) or IL-25 and TSLP (R\&D Systems, Minneapolis, MN) in a volume of $50 \mu \mathrm{l} \mathrm{PBS/}$ mouse for $24 \mathrm{~h}$. Anti-IL-9 antibody (R\&D Systems, $1 \mathrm{mg} /$ $\mathrm{kg}$, i.t.) or a control antibody IgG was injected $30 \mathrm{~min}$ before SS or CLP surgery.

\section{ILC2 isolation and sorting from lung tissue}

ILC2 isolation and sorting from lung tissue was performed as described previously ${ }^{27}$. Briefly, lungs were perfused with $10 \mathrm{ml}$ PBS through right ventricle of heart, and then filled with $1 \mathrm{ml}$ RPMI medium with Liberase TM $(50 \mu \mathrm{g} / \mathrm{ml}$ final concentration) and DNase I $(1 \mu \mathrm{g} / \mathrm{ml}$ final concentration) and digested in $5 \mathrm{ml}$ RPMI digestion medium for $30-45 \mathrm{~min}$ at $37^{\circ} \mathrm{C}$ with vortexing every 10 min. The resultant samples were mashed by $70 \mu \mathrm{m}$ cell strainers, washed with Dulbecco's modified Eagle media [DMEM; supplemented with $10 \%$ fetal bovine serum (FBS) and $1 \%$ penicillin/streptomycin (Thermo Fisher Scientific, Pittsburgh, PA)], and the remaining red blood cells were lysed. Cell suspensions were used for subsequent flow cytometry staining. For ILC2 sorting, total lung cells were stained with lineage cocktail Abs, against B220, CD3, CD4, CD5, CD8 $\alpha$, CD11b, CD11c, CD19, Gr1, TCR $\beta, \quad$ Ter-119, $\gamma \delta \mathrm{TCR}, \quad$ NK1.1, and FceR1 (eBioscience, San Diego, CA); anti-CD45 Ab (eBioscience); anti-ST2 Ab (MD Biosciences, Oakdale, $\mathrm{MN}$ ); and anti-CD90.2 $\mathrm{Ab}$ (eBioscience), at $4{ }^{\circ} \mathrm{C}$ for 30 min. ILC2 were defined as $\mathrm{Lin}^{-} \mathrm{CD} 90.2^{+} \mathrm{CD} 45^{+} \mathrm{ST} 2^{+}$and sorted by FACSAria (BD Biosciences). The average purity of ILC2 is $>98 \%$.

\section{MLEC isolation and culture}

MLECs were isolated as described previously ${ }^{42}$. Briefly, mice were anesthetized, chest cavity was opened, and blood was removed by infusing $10 \mathrm{ml}$ PBS. Lung tissues were diced into $\sim 1 \mathrm{~mm}^{3}$ pieces and cultured in a $60 \mathrm{~mm}$ culture dish in growth medium (DMEM containing $2 \mathrm{mM}$ glutamine, $10 \% \mathrm{FBS}, 5 \%$ human serum, $50 \mu \mathrm{g} / \mathrm{ml}$ penicillin/streptomycin, $5 \mu \mathrm{g} / \mathrm{ml}$ heparin, and $80 \mu \mathrm{g} / \mathrm{ml} \mathrm{EC}$ growth supplement from bovine brain) at $37^{\circ} \mathrm{C}$ with $5 \%$ $\mathrm{CO}_{2}$ for $60 \mathrm{~h}$. Then, remove tissue dices from dish and culture the adherent cells for 3 days. Purify the MLEC by biotin-conjugated rat anti-mouse $\mathrm{CD} 31 \mathrm{mAb}$ and $\mathrm{BD}$ IMag streptavidin particles plus-DM, and the immunomagnetic separation system (BD Biosciences) following the manufacturer's instructions. Purified MLECs were characterized by their cobblestone morphology.

\section{Lung permeability assay}

Lung vascular permeability was measured by EBD leakage in lung alveolus ${ }^{43,44}$. EBD $(20 \mathrm{mg} / \mathrm{kg}$; SigmaAldrich, St. Louis, MO) in $100 \mu \mathrm{l}$ volume was 
administered intravenously through tail vein $1 \mathrm{~h}$ before animal euthanasia. Lungs were then perfused with PBS through right ventricle to remove intravascular dye. Remove and photograph the lungs. Then the lungs were dried at $60^{\circ} \mathrm{C}$ for $48 \mathrm{~h}$ and incubated in formamide (Sigma-Aldrich) at $37^{\circ} \mathrm{C}$ for $24 \mathrm{~h}$ and centrifuged at $5000 \times g$ for $30 \mathrm{~min}$. EBD in the supernatant was measured by spectrophotometric method (wavelength 620 and $740 \mathrm{~nm}$ ). The extravasated EBD concentration was calculated according to a standard curve and expressed as the dye incorporated per mg of tissue.

\section{Flow cytometry}

Cell suspensions were stained with a combination of the following monoclonal fluorescently conjugated antibodies: fluorescein isothiocyanate-conjugated B220 (RA36B2, eBioscience), CD3 (17A2, eBioscience), CD4 (RM4-5, eBioscience), CD5 (53-7.3, eBioscience), CD8 $\alpha$ (53-6.7, eBioscience), CD11b (M1/70, eBioscience), CD11c (N418, eBioscience), CD19 (eBio1D3, eBioscience), Gr-1 (RB68C5, eBioscience), TCR $\beta$ (N57-597, eBioscience), Ter-119 (Ter119, eBioscience), $\gamma \delta \mathrm{TCR}$ (eBioGL3, eBioscience), NK1.1 (PK136, eBioscience), and FceR1 (MAR-1, eBioscience); phycoerythrin (PE)-conjugated ST2 (DJ8, MD Biosciences); allophycocyanin-conjugated CD90.2 (53-2.1, eBioscience); Alexa Fluor 700-conjugated CD45 (30-F11, eBioscience); PE-Cyanine 7-conjugated KLRG1 (2F1, BD Biosciences); and BV421-conjugated-Ly-6A/E (D7, BD Biosciences). To stain for intracellular murine antigens, cells were first stained for surface antigens, then fixed and permeabilized with intracellular fixation and permeabilization buffer set (eBioscience), according to the manufacturer's recommendations. Cells were then incubated with specific antibodies to IL-4-PE-Cyanine 7 (11B11, eBioscience), IL-9-Percp-Cy5.5 (D9302C12, BD Biosciences), and IL-13-eFluor 450 (eBio13A, eBioscience). For intracellular staining, cells were incubated with Phorbol 12-myristate 13-acetate (PMA) (50 $\mathrm{ng} / \mathrm{ml})$, ionomycin $(500 \mathrm{ng} / \mathrm{ml})$ and brefeldin $\mathrm{A}(1 \mu \mathrm{g} / \mathrm{ml})$ for $4 \mathrm{~h}$. Cells were then washed in PBS and re-suspended. Cells were analyzed by BD LSRII flow cytometer (BD Bioscences) and FlowJo software.

\section{RNA isolation and real-time quantitative PCR}

Total RNA from lung tissue was isolated by TRIZOL reagent (Life Technologies, Pittsburgh, PA) according to the manufacturer's instructions. cDNA was generated using iScript Reverse Transcription Supermix (Bio-Rad, Hercules, CA). Quantitative real-time PCR was performed using SYBR Green Supermix (Bio-Rad) and run in a BioRad iQ5 real-time PCR machine. All data were normalized to $18 \mathrm{~s}$ values, analyzed using comparative CT method and presented as the fold-increase over WT controls.

\section{Enzyme-linked immunosorbent assay}

Cytokine and chemokine concentrations in plasma, BALF, and PLF were determined by IL-9 and IL-13 enzyme-linked immunosorbent assay (eBioscience) according to each manufacturer's instructions.

\section{MLEC immunofluorescence staining}

For cell death measurement, purified MLECs were stained with Alexa Fluor 488-labeled FLICA (ImmunoChemistry, Bloomington, MN), and TUNEL staining (Roche Applied Science, Indianapolis, IN), nuclear staining with $1 \mu \mathrm{g} / \mathrm{ml}$ Hoechst 33258 (Sigma-Aldrich). We captured images with FV1000 (Olympus). Each slide were chosen at least five randomly fields and analyzed in duplicate individual experiments.

\section{Western blotting}

MLEC lysates were separated by 5 and $10 \%$ SDSpolyacrylamide gel electrophoresis, and then were transferred onto polyvinylidene difluoride membranes. The membranes were blocked with blocking buffer (LI-COR Biosciences, Lincoln, NE) for $1 \mathrm{~h}$ at room temperature and incubated with the anti-caspase-1 antibody (Abcam, Cambridge, MA) at $4{ }^{\circ} \mathrm{C}$ overnight. After washing, membranes were incubated with secondary antibodies (LICOR Biosciences) for $1 \mathrm{~h}$. Protein bands were visualized using the Odyssey System (LI-COR Biosciences).

\section{Statistical analysis}

GraphPad Software was used to analyze data by twotailed unpaired Student's $t$-tests or one-way analysis of variance tests. No randomization was used in these studies. Data are presented as mean \pm SEM. $P<0.05$ was considered statistically significant $\left({ }^{*} P<0.05\right.$; $\left.{ }^{* *} P<0.01\right)$.

\begin{abstract}
Acknowledgements
This work was supported by the National Institutes of Health Grant R01-HL079669 (J.F. and M.A.W.), National Institutes of Health Grant R56-HL-123882 (J. F.), National Institutes of Health Grant R01HL076179-09 (J.F.), VA Merit Award 1101 BX002729 (J.F.), National Natural Science Foundation of China 81470262 (J. F.), Zhejiang Provincial Program for the Cultivation of High-level Innovative Health talents 2016-6 (Q.S.), the National Natural Science Foundation of China 81671956 (Q.S.), and National Institutes of Health Grant R01GM102146 (M.J.S).
\end{abstract}

\section{Authors' contributions}

D.L., J.T., L.C., E.K.F., and Y.L. planned and did experiments, including cell isolation and treatment, confocal microscopy, and flow cytometry; D.L., J.T., and L.C. did animal experiments; D.L., Y.L., T.R.B., M.A.W., X.F., Q.S., and J.F. planned the project and conceived the experiments; D.L., E.K.F., M.J.S., X.F., Q.S., and J.F. conceived the data and wrote the manuscript. All authors read and approved the final manuscript.

\section{Author details}

'Department of Thoracic and Cardiovascular Surgery, The Children's Hospital of Zhejiang University School of Medicine, Hangzhou, Zhejiang, China.

${ }^{2}$ Department of Surgery, University of Pittsburgh School of Medicine, Pittsburgh, PA, USA. ${ }^{3}$ Department of Anesthesiology, Nanfang Hospital, Southern Medical University, Guangzhou, China. ${ }^{4}$ Department of Thoracic Surgery, Shanghai Pulmonary Hospital, Tongji University School of Medicine, 
Shanghai, China. ${ }^{5}$ University of Pittsburgh School of Arts and Science, Pittsburgh, PA, USA. ${ }^{6}$ Research and Development, Veterans Affairs Pittsburgh Healthcare System, Pittsburgh, PA, USA. ${ }^{7}$ McGowan Institute for Regenerative Medicine, University of Pittsburgh, Pittsburgh, PA, USA. ${ }^{8}$ Department of Anesthesiology and Intensive Care Unit, The First Affiliated Hospital, Zhejiang University School of Medicine, Hangzhou, Zhejiang, China

\section{Conflict of interest}

The authors declare that they have no conflict of interest.

\section{Publisher's note}

Springer Nature remains neutral with regard to jurisdictional claims in published maps and institutional affiliations.

Supplementary Information accompanies this paper at (https://doi.org/ 10.1038/s41419-018-0412-5).

Received: 11 December 2017 Revised: 13 February 2018 Accepted: 15 February 2018

Published online: 06 March 2018

\section{References}

1. Singer, M. et al. The Third International Consensus Definitions for Sepsis and Septic Shock (Sepsis-3). JAMA 31, 801-810 (2016).

2. Kaukonen, K. M., Bailey, M., Suzuki, S., Pilcher, D. \& Bellomo, R. Mortality related to severe sepsis and septic shock among critically ill patients in Australia and New Zealand, 2000-2012. JAMA 311, 1308-1316 (2014).

3. Ani, C., Farshidpanah, S., Bellinghausen Stewart, A. \& Nguyen, H. B. Variations in organism-specific severe sepsis mortality in the United States: 1999-2008. Crit. Care Med. 43, 65-77 (2015).

4. Fleischmann, $\mathbf{C}$. et al. Assessment of global incidence and mortality of hospital-treated sepsis. Current estimates and limitations. Am. J. Respir. Crit. Care Med. 193, 259-272 (2016).

5. Angus, D. C. \& van der Poll, T. Severe sepsis and septic shock. N. Engl. J. Med. 369, 840-851 (2013).

6. Trzeciak, S. et al. Early microcirculatory perfusion derangements in patients with severe sepsis and septic shock: relationship to hemodynamics, oxygen transport, and survival. Ann. Emerg. Med. 49, 88-98 (2007) (98 e81-82).

7. Edul, V. S. et al. Quantitative assessment of the microcirculation in healthy volunteers and in patients with septic shock. Crit. Care Med. 40, 1443-1448 (2012).

8. Andonegui, G. et al. Mice that exclusively express TLR4 on endothelial cells can efficiently clear a lethal systemic Gram-negative bacterial infection. J. Clin. Invest. 119, 1921-1930 (2009).

9. Gill, S. E., Rohan, M. \& Mehta, S. Role of pulmonary microvascular endothelial cell apoptosis in murine sepsis-induced lung injury in vivo. Respir. Res. 16, 109 (2015).

10. Liang, Y. et al. Elevated levels of plasma TNF-alpha are associated with microvascular endothelial dysfunction in patients with sepsis through activating the NF-kappaB and p38 mitogen-activated protein kinase in endothelial cells. Shock 41, 275-281 (2014).

11. $\mathrm{Xi}, \mathrm{H}$. et al. Caspase-1 inflammasome activation mediates homocysteineinduced pyrop-apoptosis in endothelial cells. Circ. Res. 118, 1525-1539 (2016).

12. Bergsbaken, T., Fink, S. L. \& Cookson, B. T. Pyroptosis: host cell death and inflammation. Nat. Rev. Microbiol. 7, 99-109 (2009).

13. Fink, S. L. \& Cookson, B. T. Caspase-1-dependent pore formation during pyroptosis leads to osmotic lysis of infected host macrophages. Cell. Microbiol. 8 1812-1825 (2006).

14. Yang, J. et al. Hemorrhagic shock primes for lung vascular endothelial cell pyroptosis: role in pulmonary inflammation following LPS. Cell Death Dis. 7, e2363 (2016).

15. Spits, H. et al. Innate lymphoid cells-a proposal for uniform nomenclature Nat. Rev. Immunol. 13, 145-149 (2013).

16. Sonnenberg, G. F. \& Artis, D. Innate lymphoid cells in the initiation, regulation and resolution of inflammation. Nat. Med. 21, 698-708 (2015).

17. Monticelli, L. A. et al. Innate lymphoid cells promote lung-tissue homeostasis after infection with influenza virus. Nat. Immunol. 12, 1045-1054 (2011).
18. Sugita, K. et al. Type 2 innate lymphoid cells disrupt bronchial epithelial barrier integrity by targeting tight junctions Via IL-13 in Asthma. J. Allergy Clin Immunol. 141, 300-310 e311 (2017).

19. Chang, Y. J. et al. Innate lymphoid cells mediate influenza-induced airway hyper-reactivity independently of adaptive immunity. Nat. Immunol. 12, 631-638 (2011).

20. Mjosberg, J. M. et al. Human IL-25- and IL-33-responsive type 2 innate lymphoid cells are defined by expression of CRTH2 and CD161. Nat. Immunol. 12 1055-1062 (2011).

21. Huang, Y. et al. IL-25-responsive, lineage-negative KLRG1(hi) cells are multipotential 'inflammatory' type 2 innate lymphoid cells. Nat. Immunol. 16 161-169 (2015).

22. Kim, B. S. et al. TSLP elicits IL-33-independent innate lymphoid cell responses to promote skin inflammation. Sci. Transl. Med. 5, 170 ra116 (2013).

23. Gasteiger, G., Fan, X., Dikiy, S., Lee, S. Y. \& Rudensky, A. Y. Tissue residency of innate lymphoid cells in lymphoid and nonlymphoid organs. Science 350, 981-985 (2015).

24. Nascimento, D. C. et al. IL-33 contributes to sepsis-induced long-term immunosuppression by expanding the regulatory $\mathrm{T}$ cell population. Nat. Commun. 8, 14919 (2017)

25. Rittirsch, D., Huber-Lang, M. S., Flierl, M. A. \& Ward, P. A. Immunodesign of experimental sepsis by cecal ligation and puncture. Nat. Protoc. 4, 31-36 (2009).

26. Seehus, C. R. et al. The development of innate lymphoid cells requires TOX dependent generation of a common innate lymphoid cell progenitor. Nat. Immunol. 16, 599-608 (2015)

27. Moro, K., Ealey, K. N., Kabata, H. \& Koyasu, S. Isolation and analysis of group 2 innate lymphoid cells in mice. Nat. Protoc. 10, 792-806 (2015).

28. Neill, D. R. et al. Nuocytes represent a new innate effector leukocyte that mediates type-2 immunity. Nature 464, 1367-1370 (2010).

29. Zhang, W. et al. IL-9 aggravates the development of atherosclerosis in ApoE -/- mice. Cardiovasc. Res. 106, 453-464 (2015).

30. Mohapatra, A. et al. Group 2 innate lymphoid cells utilize the IRF4-IL-9 module to coordinate epithelial cell maintenance of lung homeostasis. Mucosal Immunol. 9, 275-286 (2016).

31. Richard, M., Grencis, R. K., Humphreys, N. E., Renauld, J. C. \& Van Snick, J. Anti-IL9 vaccination prevents worm expulsion and blood eosinophilia in Trichuris muris-infected mice. Proc. Natl Acad. Sci. USA 97, 767-772 (2000).

32. Rauber, S. et al. Resolution of inflammation by interleukin-9-producing type 2 innate lymphoid cells. Nat. Med. 23, 938-944 (2017).

33. Turner, J. E. et al. IL-9-mediated survival of type 2 innate lymphoid cells promotes damage control in helminth-induced lung inflammation. J. Exp. Med. 210, 2951-2965 (2013).

34. Wilhelm, C. et al. An IL-9 fate reporter demonstrates the induction of an innate IL-9 response in lung inflammation. Nat. Immunol. 12, 1071-1077 (2011).

35. Choi, Y. S. et al. Interleukin-33 induces angiogenesis and vascular permeability through ST2/TRAF6-mediated endothelial nitric oxide production. Blood 114, 3117-3126 (2009)

36. Gadani, S. P., Smirnov, I., Smith, A. T., Overall, C. C. \& Kipnis, J. Characterization of meningeal type 2 innate lymphocytes and their response to CNS injury. J. Exp. Med. 214, 285-296 (2017).

37. Coletta, C. et al. Endothelial dysfunction is a potential contributor to multiple organ failure and mortality in aged mice subjected to septic shock: preclinical studies in a murine model of cecal ligation and puncture. Crit. Care 18, 511 (2014).

38. Gill, S. E., Taneja, R., Rohan, M., Wang, L. \& Mehta, S. Pulmonary microvascular albumin leak is associated with endothelial cell death in murine sepsisinduced lung injury in vivo. PLOS ONE 9, e88501 (2014).

39. Aird, W. C. The role of the endothelium in severe sepsis and multiple organ dysfunction syndrome. Blood 101, 3765-3777 (2003).

40. Shi, J. et al. Cleavage of GSDMD by inflammatory caspases determines pyroptotic cell death. Nature 526, 660-665 (2015).

41. Miao, E. A., Rajan, J. V. \& Aderem, A. Caspase-1-induced pyroptotic cell death Immunol. Rev. 243, 206-214 (2011).

42. Xiang, M. et al. Hemorrhagic shock activation of NLRP3 inflammasome in lung endothelial cells. J. Immunol. 187, 4809-4817 (2011).

43. Radu, M. \& Chernoff, J. An in vivo assay to test blood vessel permeability. J. Vis. Exp., e50062 (2013).

44. Yang, W. L. et al. Cold-inducible RNA-binding protein causes endothelia dysfunction via activation of Nlrp3 inflammasome. Sci. Rep. 6, 26571 (2016). 